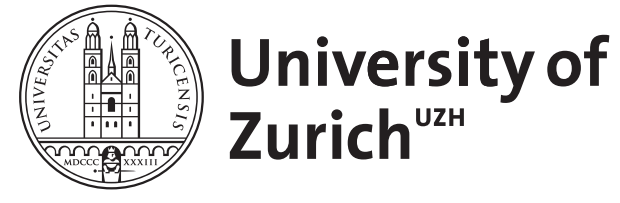

\title{
Cardiac hybrid imaging: state-of-the-art
}

\author{
Kaufmann, P A
}

\begin{abstract}
The field of noninvasive cardiac imaging has experienced enormous advances including computerized tomography coronary angiography (CTCA). Invasive angiography remains the anatomic standard of reference but it is associated with a non-negligible peri-procedural morbidity and mortality which suggests confining its use to patients who will benefit from a revascularization procedure. Many factors that are beyond the simple quantification of diameter narrowing and therefore cannot be fully assessed with luminology will eventually determine whether or not a given lesion produces stress-induced ischemia. Myocardial perfusion scintigraphy by single photon emission computerized tomography (SPECT) is one of the most widely used and well established noninvasive tools for the diagnosis of ischemic heart disease. Although positron emission tomography (PET) offers a higher accuracy than SPECT its use is often limited to large centers. This article explains the great potential of cardiac hybrid imaging which allows a comprehensive evaluation of coronary artery disease as it combines both morphological and functional information by fusing either SPECT or PET with CTCA. SPECT/CT and PET/CT hybrid imaging can provide entirely noninvasively unique information which helps improving diagnostic assessment and risk stratification and also impacts decision making with regard to revascularization in patients with coronary artery disease.
\end{abstract}

DOI: https://doi.org/10.1007/s12149-009-0245-5

Posted at the Zurich Open Repository and Archive, University of Zurich

ZORA URL: https://doi.org/10.5167/uzh-18682

Journal Article

Published Version

Originally published at:

Kaufmann, P A (2009). Cardiac hybrid imaging: state-of-the-art. Annals of Nuclear Medicine, 23(4):325331.

DOI: https://doi.org/10.1007/s12149-009-0245-5 


\title{
Cardiac hybrid imaging: state-of-the-art
}

\author{
Philipp A. Kaufmann
}

Received: 15 January 2009/Accepted: 18 January 2009/Published online: 10 April 2009

(C) The Japanese Society of Nuclear Medicine 2009

\begin{abstract}
The field of noninvasive cardiac imaging has experienced enormous advances including computerized tomography coronary angiography (CTCA). Invasive angiography remains the anatomic standard of reference but it is associated with a non-negligible peri-procedural morbidity and mortality which suggests confining its use to patients who will benefit from a revascularization procedure. Many factors that are beyond the simple quantification of diameter narrowing and therefore cannot be fully assessed with luminology will eventually determine whether or not a given lesion produces stress-induced ischemia. Myocardial perfusion scintigraphy by single photon emission computerized tomography (SPECT) is one of the most widely used and well established noninvasive tools for the diagnosis of ischemic heart disease. Although positron emission tomography (PET) offers a higher accuracy than SPECT its use is often limited to large centers. This article explains the great potential of cardiac hybrid imaging which allows a comprehensive evaluation of coronary artery disease as it combines both morphological and functional information by fusing either SPECT or PET with CTCA. SPECT/CT and PET/CT hybrid imaging can provide entirely noninvasively unique information which helps improving diagnostic assessment and risk stratification and
\end{abstract}

The University Hospital Zurich holds a research contract with GE Healthcare (Milwaukee, WI, USA).

P. A. Kaufmann $(\square)$

Cardiac Imaging, University Hospital Zurich,

NUK C 42, Raemistrasse 100, 8091 Zurich, Switzerland

e-mail: pak@usz.ch

P. A. Kaufmann

Zurich Center for Integrative Human Physiology (ZIHP),

University of Zurich, Zurich, Switzerland also impacts decision making with regard to revascularization in patients with coronary artery disease.

Keywords Hybrid cardiac imaging - SPECT/CT . $\mathrm{PET} / \mathrm{CT} \cdot$ Nuclear cardiology

\section{Coronary stenoses and ischemic heart disease: two faces of the same coin}

Despite the trend in modern medicine away from intervention towards prevention the treatment of coronary artery disease has remained of great importance in industrialized countries. Although invasive coronary angiography is affected by a considerable morbidity (1.5\%) [1] and mortality $(0.3 \%)$ [2] this has remained the most established method for visualization of the coronary arteries and, thus, the standard of reference for the diagnosis of coronary stenoses. It is, however, far from being a perfect diagnostic tool as in fact its accuracy is severely hampered by a large intraobserver and interobserver variability in defining the anatomic relevance of stenoses (up to 50\%) [3, 4]. This is further underlined by the fact that invasive coronary angiography is very poorly correlated with postmortem coronary anatomy [5, 6]. Most importantly, angiographic findings are poor predictors of physiologic relevance of a coronary stenosis $[4,7-10]$. A substantial fraction-namely between 20 and $40 \%$ - of all diagnostic invasive coronary angiograms reveal clinically insignificant disease [1]. This has lead to a search for strategies for non-invasive coronary imaging, among them dichromatic synchrotron radiation [11], electron beam tomography [12], magnetic resonance imaging [13, 14], and multidetector computerized tomography coronary angiography (CTCA) [15]. Only the latter has recently emerged as an acceptable alternative to the 
invasive coronary angiography for visualization of the coronary anatomy.

It is very likely, but still awaits confirmation in large multicenter trials, that CTCA will help to change the fact that at present only about a third of patients referred to catheterization will eventually undergo a revascularization procedure [16] leaving most of the patients exposed to the risks but without the benefit of a catheter in their coronary arteries. Coronary anatomy, however, may not allow to estimate with certainty the pathophysiologic relevance of a coronary lesion, as particularly in intermediate lesions there are many factors influencing the interrelation between anatomic finding and hemodynamic consequences which cannot be fully elucidated by anatomic evaluation alone, not even with the use of quantitative coronary angiography [17]. Therefore, according to the actual European and American guidelines a test for ischemia before any elective coronary angiography is mandatory [18-20]. It appears, however, that there is still a large gap between these evidence-based guidelines and the daily clinical routine, as underlined by a recent study by Lin et al. reporting that in a large cohort of patients $(n=23887)$ referred for elective coronary intervention only a minority (44\%) had undergone any kind of stress testing in a 90-day period prior to the intervention [21]. This impressively underlines the need to increase both the awareness of the importance and the availability of non-invasive testing for coronary artery disease.

\section{Role of single photon emission computerized tomography (SPECT)}

Nuclear myocardial perfusion imaging (MPI) with SPECT represents the most widely available, robust and by far best established non-invasive method for this purpose [22], while coronary angiography has remained the standard of reference for the anatomy of the epicardial coronary artery anatomy. A perfect agreement of MPI SPECT and coronary angiography cannot be expected and is not a main prerequisite for its clinical value. In the non-invasive diagnostic algorithm the main role of SPECT is not to correctly predict or exclude epicardial coronary lesions but rather to evaluate the physiological relevance of known or suspected coronary stenoses. Nevertheless, as invasive coronary angiography is the generally accepted standard of reference for coronary lesions the findings of functional tests including SPECT have often been compared to those of coronary angiography. The results may depend at least in part from the size and selection of the study population. One of the largest recent reports, the British ROBUSTstudy, included 2560 patients who were randomized to one of the commonly used tracers (Thallium, Sestamibi or
Tetrofosmin) applying mainly adenosine stress. The authors found a sensitivity of $91 \%$ and a specificity of $87 \%$ without differences between the tracers [23].

\section{Role of positron emission tomography (PET)}

The most important perfusion tracers for clinical PET are $\mathrm{N}$-13-ammonia and-mainly in the US-Rubidium-82. Several studies involving over 1400 patients have recently been included in a meta-analysis which confirmed the high sensitivity and specificity of PET MPI. The use of Rubidium-82 offers the advantage that there is no need of any cyclotron. By contrast, the application of N-13-ammonia and alternatively of $\mathrm{O}-15$-water requires an on-site cyclotron due to the short half lives (i.e. $10 \mathrm{~min}$ and $2 \mathrm{~min}$, respectively). This major drawback seems to be responsible for the fact that this technique has not gained wide acceptance in the clinical arena. Theoretically, O-15-water may appear to be the ideal myocardial perfusion tracer as it diffuses almost freely through cell membranes. Unfortunately it does not allow achieving a strong signal-to-noise ratio between the myocardium and the ventricular blood pool due to the lack of accumulation into the myocytes which precludes obtaining clinically meaningful perfusion images. Therefore, application of O-15-water can only provide useful information if postprocessing with quantitative evaluation is performed. As this is a relative complex procedure O-15-water has mainly remained confined to scientific applications [24]. As long as an on-site cyclotron is available $\mathrm{N}$-13-ammonia has remained the perfusion tracer of choice because it provides high-quality perfusion images for visual analysis for daily clinical routine and at the same time also allows quantification of global and regional myocardial perfusion. Because PET confers a higher resolution than SPECT and inherently uses attenuation correction there is a general expert consensus that sensitivity and specificity of PET is superior to that of SPECT although this perception is not based on a large number of patients studied in head-to-head comparisons between SPECT and PET. Finally, it remains to be elucidated whether the higher costs of PET will be justified by the improvement of accuracy compared to SPECT.

\section{Definition and general aspects of hybrid imaging}

The consideration of the duality of morphology and function has gained importance over the last few years. This has lead to the conviction that comprehensive assessment of coronary disease should include both, information on coronary lesion morphology and on myocardial perfusion. Noninvasive assessment of myocardial perfusion scintigraphy 
has been available for more than three decades. By contrast non-invasive coronary angiography has only been introduced recently into the clinical arena after the advent of multi-detector computed tomography [15]. The latter technology has finally met the requirements with regard to temporal and spatial resolution to allow clinical use. This has provided the base for combination of CTCA with complementary nuclear modalities into hybrid imaging. In addition to the developments in non-invasive CTCA advances in image processing software (Fig. 1a, b) [25] and the introduction of hybrid scanners have paved the way for integrating image data sets from different modalities into hybrid images. Such software reliably allows superposition of myocardial segments depicted by SPECT or PET onto cardiac CT anatomy, resulting in an easily interpretable panoramic view of the heart, which integrates the highresolution 3-dimensional information of the coronary arteries with the functional information of the SPECT perfusion image (Fig. 1c, d). Despite the integration of highend CT devices with the capability to perform state-of-theart coronary CT angiography into nuclear scanners to form dedicated cardiac hybrid scanners, manual image coregistration may remain indispensable. Studies with X-ray based attenuation correction have reported that automated coregistration of CT and SPECT images is often unreliable and manual correction for misalignment is needed in the vast majority of the cases [26, 27]. Dedicated cardiac fusion software packages are now commercially available allowing software-based hybrid imaging with an excellent interobserver reproducibility and short processing durations [25].
Although fusion of invasive coronary angiography with SPECT has been attempted in the past, the warping and 3dimensional unification by forcing a planar 2-dimensional angiogram into a fusion with a 3-dimensional perfusion scan data set provided technically unsatisfying results. In addition, such approach precludes non-invasive preplanning of the intervention as the information on the coronary anatomy is obtained by invasive coronary angiography. During such procedure, however, rapid decision making should not be delayed by the need of time consuming offline analyses. This drawback may explain why this concept which does not allow careful non-invasive planning of the elective intervention has not been adopted into daily clinical routine.

At this point it should be clarified that the definition of hybrid imaging is the combination and fusing of two imaging data stets by which both modalities equally contribute to image information [28]. This is important as the term hybrid imaging has also been used in different contexts potentially raising some confusion about its exact meaning. For example, some authors have used the term hybrid imaging for X-ray based attenuation correction although in such setting the CT images do not provide neither anatomical nor functional information but rather exclusively contribute to improve image quality of the other modality (PET or SPECT). In fact, the parametric maps obtained from low-dose CT do not provide image information beyond that needed for attenuation correction $[26,29]$. This is underlined by the fact that Ge-68 sources used in the previous generation of PET scanners provided
Fig. 1 Illustration of the main software fusion process including a image coregistration, $\mathbf{b}$ epicardial contour detection, $\mathbf{c}$ coronary artery segmentation, and d 3-dimensional volume rendered fusion
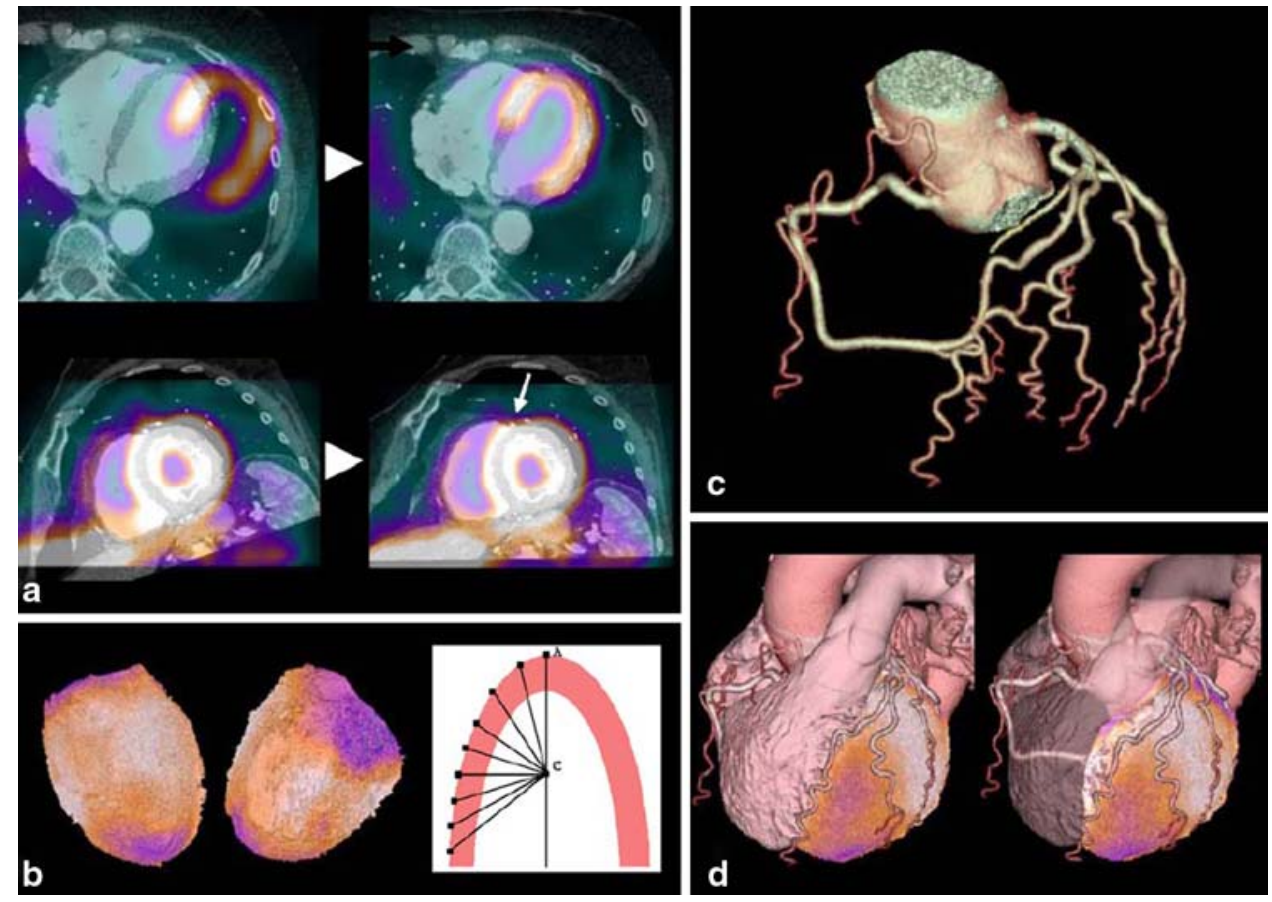
the same information, but such type of imaging was not perceived as hybrid imaging as attenuation correction does not contribute to topographic image information. Others have used the term "hybrid imaging" for the side-by-side analysis of MPI and CT images [30]. To avoid confusion, we suggest using the term hybrid imaging for any combination of structural and functional information beyond that offered by attenuation correction or side-by-side analysis, i.e. fusion of two separate data sets into one image (Fig. 1d). Thus, this definition would not include attenuation corrected images without integrating anatomical information. Similarly, separate acquisition of structural information as well as functional (for example perfusion) data on two separate scanners or on one hybrid device would allow mental integration of side-by-side evaluation but only fusion of both pieces of information would result in a hybrid image.

\section{Role of hybrid imaging: implications and limitations}

The continuing rapid evolution of CTCA suggests that, when combined with perfusion imaging, it has the potential to be implemented into clinical practice. As mentioned above the advancements in fast-processing software for 3dimensional reconstructions have allowed initial promising attempts of purely noninvasive CAD assessment directly relating individual myocardial wall territories with impaired myocardial perfusion to the subtending coronary artery by use of PET and CT [31]. Recent data using SPECT and CT suggest that hybrid imaging provides added diagnostic clinical value beyond that of either technique alone or that of side-by-side analysis [28, 32, 33]. The added value seems to be found on both sides of the diagnostic spectrum, i.e. in low risk populations [34] as well as in those with multi-vessel disease [33]. In low risk populations hybrid imaging may increase the confidence to rule out coronary artery disease for example in the stepwise evaluation of coronary artery disease when the first study has yielded equivocal results and a second modality is needed to finally rule out with certainty any disease. Many of those patients would end up with invasive coronary angiography while hybrid imaging increases diagnostic confidence by avoiding equivocal findings which helps to reduce the number of patients unnecessarily exposed to the non-negligible risks of morbidity and mortality [2] associated with invasive coronary procedures. A the other end of the spectrum, i.e. in patients who are older then those mentioned above and who often suffer from multivessel disease with more jeopardized myocardium, hybrid imaging provides important comprehensive information to allow for timely and appropriate treatment. In such setting the value of hybrid imaging lies far beyond the simple addition of a further diagnostic test as it allows accurate spatial association of perfusion defects to their subtending coronary stenosis (Fig. 2; [35]). Hybrid imaging is a completely non-invasive approach to coronary artery disease which allows obtaining important information prior to any invasive procedure and provides the detailed information for evidence driven intervention targeting relevant lesion only. Although the CT part of hybrid imaging has excellent abilities in ruling out hemodynamic relevant coronary artery disease, an abnormal CTCA-like an abnormal conventional angiography study-is a poor predictor of ischemia, and further perfusion imaging testing is warranted to identify those patients who might benefit from a revascularization procedure [36, 37] and those in whom conservative management and risk modification may be justified [38]. Conversely, a normal MPI result does not exclude the presence of subclinical coronary disease as assessable with CTCA and conventional coronary angiography for which aggressive cardiovascular risk modification may be warranted. The incongruence of CTCA and MPI is inherent to the duality of morphologic versus functional testing. The technologic refinements implemented in the latest CT scanner generations have reduced the number of non-evaluable coronary segments and further improvements may be expected. However, no matter how accurate CTCA will possibly get with future advances in technology, the two pieces of information obtained with perfusion imaging versus morphology are difficult to compare. This has been recently documented by Gaemperli et al. who found that the receiver operator characteristic analysis for detection of perfusion defects (by SPECT) showed similar area under the curves for the reference standard, conventional angiography, and for CT angiography, documenting comparable performance and limitations of both anatomic morphologic techniques [39]. Hence, many factors that are beyond the simple quantification of diameter narrowing and that therefore cannot be fully assessed with luminology will eventually determine whether or not a given lesion produces stress-induced ischemia.

First clinical results from our institution appear encouraging, and support that hybrid images offer superior diagnostic information with regard to identification of the culprit vessel with the hemodynamic relevant lesion and increases diagnostic confidence for categorizing intermediate lesions and equivocal perfusion defects as in almost one-third of patients the fused analysis provided added diagnostic information not obtained on side-by-side analysis [33]. The incremental value seems most pronounced for functionally relevant lesions in distal segments and diagonal branches and in vessels with extensive coronary lesions or heavy calcifications on CTCA. Other groups have confirmed the added value of hybrid cardiac imaging [40]. Results from a first multicenter study underline the 
Fig. 2 a Polar maps of myocardial perfusion imaging with $99 \mathrm{mTc}$-Tetrofosmin show a pronounced inferior basal perfusion defect at adenosine stress (upper panel) which is almost entirely reversible at rest (lower panel) indicating an ischemia. The territory of this ischemia would generally be assumed to be subtended by the right coronary artery (RCA). b Anterior view of the 3dimensional hybrid cardiac image obtained by fusion the polar maps with the volume rendered CT coronary angiography of the same patient. Despite calcifications in the left anterior descending artery there are no perfusion defects in the anterior wall. c The posterior view allows recognizing, that in this patient the ischemic basal inferior territory is subtended by the circumflex artery (CX) causing the ischemia due to a tight lesion. By contrast, the RCA does not contribute to this ischemia. (Modified with permission from [35], copyright University Hospital Zurich, Switzerland)
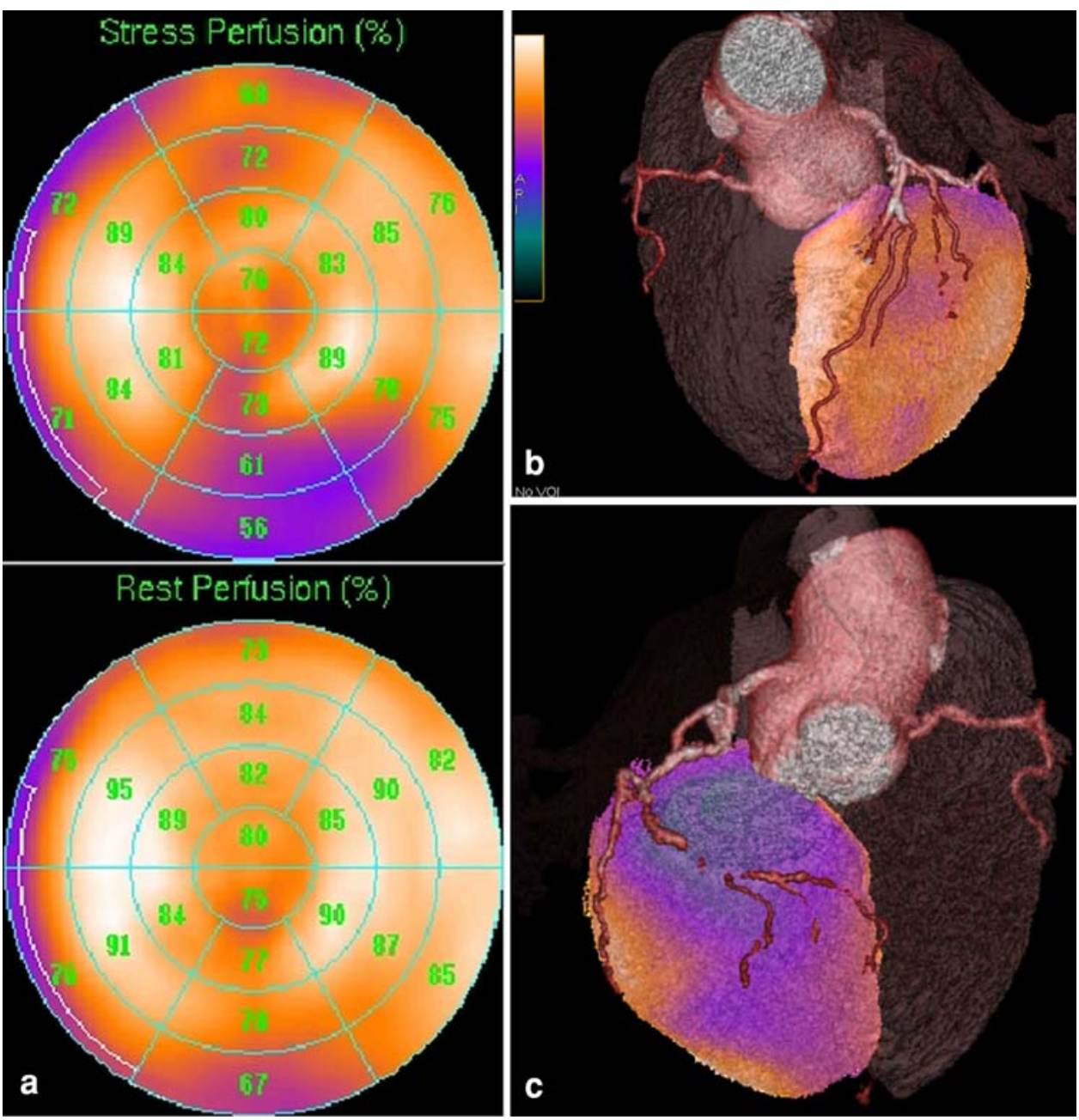

value of a combined functional and anatomical approach even without hybrid imaging showing that this combination allows improved risk stratification [41]. The clinical usefulness in terms of impact on treatment strategy and subsequently on outcome by hybrid imaging remains, however, to be determined in prospective and long-term studies. Similarly, it remains uncertain at this point whether hybrid scanners offer advantages over software fusion of data sets obtained from different scanners, as by either way one can obtain hybrid images [28]. The scan time discrepancy between emission from nuclear and CT transmission determines that high-end CT facilities constituting the CT component of hybrid cardiac scanners will be blocked by long emission scan time and is therefore forced to operate at low capacity. On the other hand, a combined device may fit into one room and needs one operating team and does not require positioning of the patient into two different scanners. The development of ultrafast SPECT scanners allowing substantially shorter acquisition time may shift the balance towards hybrid scanners in the future.
Acknowledgments Philipp A. Kaufmann is funded by a grant from the Swiss National Science Foundation (SNSF-professorship grant No. PP00A-114706).

\section{References}

1. Achenbach S, Daniel WG. Noninvasive coronary angiography-an acceptable alternative? N Engl J Med. 2001;345:1909-10.

2. Shaw LJ, Shaw RE, Merz CN, Brindis RG, Klein LW, Nallamothu B, et al. Impact of ethnicity and gender differences on angiographic coronary artery disease prevalence and in-hospital mortality in the American College of Cardiology-National Cardiovascular Data Registry. Circulation. 2008;117:1787-801.

3. Galbraith JE, Murphy ML, Desoyza N. Coronary angiogram interpretation: interobserver variability. JAMA. 1981;240:20539.

4. White CW, Wright CB, Doty DB, Hiratza LF, Eastham CL, Harrison DG, et al. Does visual interpretation of the coronary arteriogram predict the physiologic importance of a coronary stenosis? N Engl J Med. 1984;310:819-24.

5. Arnett EN, Isner JM, Redwood DR, Kent KM, Baker WP, Ackerstein $\mathrm{H}$, et al. Coronary artery narrowing in coronary heart disease: comparison of cineangiographic and necropsy findings. Ann Intern Med. 1979;91:350-6. 
6. Vlodaver Z, Frech R, Van Tassel RA, Edwards JE. Correlation of the antemortem coronary arteriogram and the postmortem specimen. Circulation. 1973;47:162-9.

7. Bech GJ, De Bruyne B, Bonnier HJ, Bartunek J, Wijns W, Peels $\mathrm{K}$, et al. Long-term follow-up after deferral of percutaneous transluminal coronary angioplasty of intermediate stenosis on the basis of coronary pressure measurement. J Am Coll Cardiol. 1998;31:841-7.

8. Pijls NH, De BB, Peels K, Van DVP, Bonnier HJ, Bartunek JKJ, et al. Measurement of fractional flow reserve to assess the functional severity of coronary-artery stenoses [see comments]. N Engl J Med. 1996;334:1703-8.

9. Topol EJ, Nissen SE. Our preoccupation with coronary luminology. The dissociation between clinical and angiographic findings in ischemic heart disease. Circulation. 1995;92:2333-42.

10. Zijlstra F, van OJ, Reiber JH, Serruys PW. Does the quantitative assessment of coronary artery dimensions predict the physiologic significance of a coronary stenosis? Circulation. 1987;75:115461.

11. Dill T, Job H, Dix WR, Ventura R, Kupper W, Hamm CW, et al. Intravenous coronary angiography with synchrotron radiation. Z Kardiol. 2000;89(Suppl 1):27-33.

12. Achenbach S, Moshage W, Ropers D, Nossen J, Daniel WG. Value of electron-beam computed tomography for the noninvasive detection of high-grade coronary-artery stenoses and occlusions. N Engl J Med. 1998;339:1964-71.

13. Kim WY, Danias PG, Stuber M, Flamm SD, Plein S, Nagel E, et al. Coronary magnetic resonance angiography for the detection of coronary stenoses. N Engl J Med. 2001;345:1863-9.

14. Manning WJ, Li W, Edelman RR. A preliminary report comparing magnetic resonance coronary angiography with conventional angiography. N Engl J Med. 1993;328:828-32.

15. Schroeder S, Achenbach S, Bengel F, Burgstahler C, Cademartiri F, de Feyter P, et al. Cardiac computed tomography: indications, applications, limitations, and training requirements: report of a Writing Group deployed by the Working Group Nuclear Cardiology and Cardiac CT of the European Society of Cardiology and the European Council of Nuclear Cardiology. Eur Heart J. 2008;29:531-56.

16. Cook S, Walpoth N, Maier W, Muehlberger V, Legrand V, Milicic D, et al. Percutaneous coronary interventions in Europe 1992-2003. EuroIntervention. 2006;1:374-9.

17. Bartunek J, Sys SU, Heyndrickx GR, Pijls NH, De Bruyne B. Quantitative coronary angiography in predicting functional significance of stenoses in an unselected patient cohort. J Am Coll Cardiol. 1995;26:328-34.

18. Fox K, Garcia MA, Ardissino D, Buszman P, Camici PG, Crea F, et al. Guidelines on the management of stable angina pectoris: executive summary: the Task Force on the Management of Stable Angina Pectoris of the European Society of Cardiology. Eur Heart J. 2006;27:1341-81.

19. Klocke FJ, Baird MG, Lorell BH, Bateman TM, Messer JV, Berman DS, et al. ACC/AHA/ASNC guidelines for the clinical use of cardiac radionuclide imaging-executive summary: a report of the American College of Cardiology/American Heart Association Task Force on Practice Guidelines (ACC/AHA/ASNC Committee to Revise the 1995 Guidelines for the Clinical Use of Cardiac Radionuclide Imaging). Circulation. 2003;108:1404-18.

20. Smith SC Jr, Dove JT, Jacobs AK, Kennedy JW, Kereiakes D, Kern $\mathrm{MJ}$, et al. ACC/AHA guidelines of percutaneous coronary interventions (revision of the 1993 PTCA guidelines)-executive summary. A report of the American College of Cardiology/American Heart Association Task Force on Practice Guidelines (committee to revise the 1993 guidelines for percutaneous transluminal coronary angioplasty). J Am Coll Cardiol 2001;37:2215-39.
21. Lin GA, Dudley RA, Lucas FL, Malenka DJ, Vittinghoff E, Redberg RF. Frequency of stress testing to document ischemia prior to elective percutaneous coronary intervention. JAMA. 2008;300:1765-73.

22. Underwood SR, Anagnostopoulos C, Cerqueira M, Ell PJ, Flint EJ, Harbinson M, et al. Myocardial perfusion scintigraphy: the evidence. Eur J Nucl Med Mol Imaging. 2004;31:261-91.

23. Kapur A, Latus KA, Davies G, Dhawan RT, Eastick S, Jarritt PH, et al. A comparison of three radionuclide myocardial perfusion tracers in clinical practice: the ROBUST study. Eur J Nucl Med Mol Imaging. 2002;29:1608-16.

24. Kaufmann PA, Camici PG. Myocardial blood flow measurement by PET: technical aspects and clinical applications. J Nucl Med. 2005;46:75-88.

25. Gaemperli O, Schepis T, Kalff V, Namdar M, Valenta I, Stefani $\mathrm{L}$, et al. Validation of a new cardiac image fusion software for three-dimensional integration of myocardial perfusion SPECT and stand-alone 64-slice CT angiography. Eur J Nucl Med Mol Imaging. 2007;34:1097-106.

26. Schepis T, Gaemperli O, Koepfli P, Ruegg C, Burger C, Leschka $\mathrm{S}$, et al. Use of coronary calcium score scans from stand-alone multislice computed tomography for attenuation correction of myocardial perfusion SPECT. Eur J Nucl Med Mol Imaging. 2007;34:11-9.

27. Goetze S, Wahl RL. Prevalence of misregistration between SPECT and CT for attenuation-corrected myocardial perfusion SPECT. J Nucl Cardiol. 2007;14:200-6.

28. Gaemperli O, Kaufmann PA. Hybrid cardiac imaging: more than the sum of its parts? J Nucl Cardiol. 2008;15:123-6.

29. Koepfli P, Hany TF, Wyss CA, Namdar M, Burger C, Konstantinidis AV, et al. CT attenuation correction for myocardial perfusion quantification using a PET/CT hybrid scanner. J Nucl Med. 2004:45:537-42.

30. Hong EC, Kimura-Hayama ET, Di Carli MF. Hybrid cardiac imaging: complementary roles of CT angiography and PET in a patient with a history of radiation therapy. J Nucl Cardiol. 2007;14:617-20.

31. Namdar M, Hany TF, Koepfli P, Siegrist PT, Burger C, Wyss CA, et al. Integrated PET/CT for the assessment of coronary artery disease: a feasibility study. J Nucl Med. 2005;46:930-5.

32. Gaemperli O, Schepis T, Kaufmann PA. SPECT-CT fusion imaging integrating anatomy and perfusion. Eur Heart J. 2007; 28:145.

33. Gaemperli O, Schepis T, Valenta I, Husmann L, Scheffel H, Duerst V, et al. Cardiac image fusion from stand-alone SPECT and CT: clinical experience. J Nucl Med. 2007;48:696-703.

34. Husmann L, Herzog BA, Gaemperli O, Tatsugami F, Burkhard N, Valenta I, et al. Diagnostic accuracy of computed tomography coronary angiography and evaluation of stress-only single-photon emission computed tomography/computed tomography hybrid imaging: comparison of prospective electrocardiogram-triggering vs. retrospective gating. Eur Heart J 2008:Dec 23 [Epub ahead of print].

35. 2006 Image of the year: focus on cardiac SPECT/CT. J Nucl Med 2006; $47: 14 \mathrm{~N}-5 \mathrm{~N}$.

36. Hachamovitch R, Hayes SW, Friedman JD, Cohen I, Berman DS. Comparison of the short-term survival benefit associated with revascularization compared with medical therapy in patients with no prior coronary artery disease undergoing stress myocardial perfusion single photon emission computed tomography. Circulation. 2003;107:2900-7.

37. Shaw LJ, Berman DS, Maron DJ, Mancini GB, Hayes SW, Hartigan PM, et al. Optimal medical therapy with or without percutaneous coronary intervention to reduce ischemic burden: results from the Clinical Outcomes Utilizing Revascularization 
and Aggressive Drug Evaluation (COURAGE) trial nuclear substudy. Circulation. 2008;117:1283-91.

38. Boden WE, O'Rourke RA, Teo KK, Hartigan PM, Maron DJ, Kostuk WJ, et al. Optimal medical therapy with or without PCI for stable coronary disease. N Engl J Med. 2007;356: 1503-16.

39. Gaemperli O, Schepis T, Valenta I, Koepfli P, Husmann L, Scheffel $\mathrm{H}$, et al. Functionally relevant coronary artery disease: comparison of 64-section CT angiography with myocardial perfusion SPECT. Radiology. 2008;248:414-23.
40. Rispler S, Keidar Z, Ghersin E, Roguin A, Soil A, Dragu R, et al. Integrated single-photon emission computed tomography and computed tomography coronary angiography for the assessment of hemodynamically significant coronary artery lesions. J Am Coll Cardiol. 2007;49:1059-67.

41. van Werkhoven JM, Schuijf JD, Gaemperli O, Jukema W, Boersma E, Wijns W, et al. Prognostic value of multi-slice computed tomography and gated single photon emission computed tomography in patients with suspected coronary artery disease. J Am Coll Cardiol. 2009;53:623-32. 